\title{
The Bibliographical Variants Between The Last of Us and The Last of Us Remastered
}

\author{
Chris J. Young
}

I

Released on 14 June 2013 exclusively for the PlayStation $3\left(\mathrm{PS}_{3}\right)$ by the studio Naughty Dog and the publisher Sony Computer Entertainment of America (SCEA), The Last of Us allows players to control Joel, and at times Ellie, as they travel across a post-apocalyptic United States. ${ }^{1}$ The game received critical acclaim from reviewers and game critics alike for its design and narrative, lauding it for expanding the boundaries of

1. When a videogame is released exclusively for a console, it is available on that console or will become available for other consoles after a specified contractual period of time between the developer and publisher of the videogame has elapsed. Naughty Dog is a first-party developer for Sony Computer Entertainment of America, which means they develop videogames exclusively for Sony's PlayStation consoles. Naughty Dog has developed eight videogame trademark properties exclusively for PlayStation consoles since they became a wholly owned subsidiary of Sony: Jak E' Daxter: The Precursor Legacy; Jak II; Jak 3; Uncharted: Drake's Fortune; Uncharted 2: Among Thieves; Uncharted 3: Drake's Deception; The Last of Us; and Uncharted 4: A Thief's End.

Chris J. Young (140 St. George Street, Toronto, ON, Canada $\mathrm{M}_{5} \mathrm{~S}_{3} \mathrm{G} 6$ ), is a PhD Candidate at the Faculty of Information, Knowledge Media Design Institute, and Semaphore Lab, University of Toronto. For his dissertation, Young is conducting a two-year ethnographic study of game makers in the Toronto game development scene. Young's research interests include games and play cultures, information seeking and use practices, cultural industry and labor, and digital humanities.

PBSA 110:3 (2016): 459-484 C 2016 Bibliographical Society of America.

All rights reserved. 0006-128X/2016/0110-0003\$10.00 


\section{$460 \quad$ Bibliographical Society of America}

videogames in digital literature. ${ }^{2}$ Upon its release, The Last of $U s$ sold over 3.4 million copies, making it the best-selling game launch of 2013 and one of the largest releases of an original intellectual property. ${ }^{3}$ The Last of $U$ s has currently sold over 7 million copies and stands as the third best-selling $\mathrm{PS}_{3}$ game of all time. ${ }^{4}$ In the year following its initial release, SCEA issued additional downloadable content (DLC) that could be purchased via the PlayStation Store (PS Store), including console themes, soundtracks, avatars, add-ons, a network pass, and a standalone game titled Left Bebind that allowed one to play as Ellie in scenes not available in the main story of The Last of $U s$.

The PlayStation $4\left(\mathrm{PS}_{4}\right)$ console was released almost six months later on 15 November 2013. Due to the widespread success of The Last of $U s$, Naughty Dog began to work on a port for the $\mathrm{PS}_{4} \cdot{ }^{5}$ In an interview with game magazine Edge, creative director Neil Druckmann explained the challenges of porting The Last of $U_{s}$ from the $\mathrm{PS}_{3}$ to the $\mathrm{PS}_{4}$.

I can't describe how difficult a task that is. And once it's running well. You're running the [versions] side by side to make sure you didn't screw something up in the process, like physics being slightly off, or lighting being shifted and all of

2. Metacritic gave The Last of $U s$ a score of 95 out of 100 based on the average score of 98 critic reviews (Metacritic Game Reviews, "The Last of Us for PlayStation 3," Metacritic [2016], accessed 25 February 2016, http://www.metacritic.com /game/playstation-3/the-last-of-us.)

3. Paul Tassi, "'The Last of Us' has the biggest launch of the year with $1.3 \mathrm{~m}$ sold," Forbes, 18 June 2013, accessed February 25, 2016, http://www.forbes.com/sites /insertcoin/2013/o6/18/the-last-of-us-is-the-biggest-game-of-the-year-with-1-3m -sold/\# 71637cb6ibbo.

4. Jason Dunning, "The Last of Us crosses 7 million sold, new DLC incoming," PlayStation Lifestyle, 16 July 2014, accessed 25 February 2016, http://www.playstation lifestyle.net/2014/o7/16/the-last-of-us-sales-figures-cross-7-million-sold-new-dlc -incoming/.

5. Porting is a process whereby a videogame developer or studio converts a videogame intended to run on one platform onto another platform. A platform is a computing hardware and/or software environment that facilitates "certain types of computational expression and innovation" (Nick Montfort and Ian Bogost, foreword, Racing the Beam: The Atari Video Computer System, [Cambridge, MA: MIT Press, 2009], vii). Porting a videogame is a widely used practice throughout the industry, with releases on multiple platforms such as PlayStation, Xbox, and Nintendo consoles, and personal computers. In the case of The Last of $U s$, Naughty Dog ported the videogame from the $\mathrm{PS}_{3}$ console to the $\mathrm{PS}_{4}$ console. 


\section{The Last of Us and The Last of Us Remastered 461}

a sudden it's a drastically different look. That's not 'improved' anymore; that's different. We want to stay faithful while being better. ${ }^{6}$

Like many developers porting original game titles to other platforms, Druckmann wanted to maintain the "work" of The Last of Us while improving its aesthetics for the recently released, and more powerful, $\mathrm{PS}_{4}$ console, redesigning the aesthetic format while retaining the original content. Naughty Dog undertook a similar approach when they ported The Last of Us to the $\mathrm{PS}_{4}$ : "visuals aside, The Last of Us Remastered is an untouched port, even going so far as to leave in common points of complaint [by players]." ${ }^{\prime \prime}$ Druckmann suggested that the primary, if not sole, difference between the $\mathrm{PS}_{3}$ and $\mathrm{PS}_{4}$ versions of the videogame is the art assets; a lengthy and time-consuming process where the developer replaces all the image files with much larger file-sizes containing increased pixilation, modified textures, lighting, and shading, completed with a new rendering.

The Last of Us Remastered 29 July 2014 release exclusively on the $\mathrm{PS}_{4}$ included the majority of the DLC released over the previous year on the $\mathrm{PS}_{3}$. Releasing later editions with similar or exclusive content has become a common practice in the videogame industry, where developers and publishers re-release "definitive," "enhanced," "high definition," or, as is the case with The Last of Us, "remastered" editions onto the same platform, or port them to another platform several months after the initial release. These re-releases are typically developed using a majority of the assets and code from the previous version to reduce the financial cost of resources and labor while releasing the title to new audiences through a different platform. Similar industry practices can be found in the film industry, where studios will re-release "high definition" editions of old films, or the book publishing industry where cheap paperbacks are printed from plates or photo-negatives used for the initial hardcover edition. Notable examples of recently re-released videogames where the assets and code have been enhanced for current videogame platforms include: Uncharted: The Nathan Drake Collection

6. "The last of it: Naughty Dog sends The Last of Us out with a definitive edition for PlayStation 4," Edge Magazine, June 2014, accessed 25 February 2016, https:/itunes.apple.com/ca/app/edge-games-magazine-for-interactive/id451410596 ?mt $=8$.

7. Ibid. 


\section{Bibliographical Society of America}

on the $\mathrm{PS}_{4}$ that includes Uncharted: Drake's Fortune, Uncharted: Among Thieves, and Uncharted: Drake's Deception from the $\mathrm{PS}_{3}$ versions (also developed by Naughty Dog); The Bioshock Collection on the PS 4 , Xbox One, and PC that includes Bioshock, Bioshock 2, and Bioshock Inifinite from the $\mathrm{PS}_{3}, \mathrm{Xbox} 360$, and PC versions; and Assassin's Creed: The Ezio Collection on $\mathrm{PS}_{4}$, Xbox One, and PC that includes Assassin's Creed II, Assassin's Creed: Brotherhood, and Assassin's Creed: Revelations from the $\mathrm{PS}_{3}$, Xbox 360, and $\mathrm{PC}$ versions. However, these re-release practices are by no means a new trend within the videogame industry. With almost every new generation of consoles there have been ports of titles aimed at current markets.

As is the case with impressions in the book publishing industry, there can also be "bibliographical variants" among videogame releases. Differences between The Last of $U s$ and The Last of Us Remastered include, but are not limited to, graphics resolution, frame-rate, gameplay, hardware, player input, and paratexts such as add-ons and other DLC associated with the respective editions. While bibliographers have developed sophisticated methods for identifying and presenting textual variants among books, we still lack similar standards for born-digital texts and electronic records. The disciplines of platform studies, software studies, computer forensics, media archaeology, and critical code studies offer methods and approaches for the analysis of hardware, software, code, and formats in born-digital texts and electronic records, but these disciplines do not directly address the questions and issues relevant to the field of bibliography. ${ }^{8}$ Challenges such as how to describe a

8. See for example: platform studies, Nick Montfort and Ian Bogost, Racing the Beam: The Atari Video Computer System (Cambridge, MA: MIT Press, 20o9), and Ian Bogost and Nick Montfort, "Platform Studies: Frequently Questioned Answers," Proceedings of the Digital Arts and Culture Conference, 2009 (12 December 2009): 6, accessed 25 February 2016, https://escholarship.org/uc/item/olrokgbr; software studies, Lev Manovich, The Language of New Media (Cambridge, MA: MIT Press, 2001), and Noah Wardrip-Fruin, Expressive Processing: Digital Fictions, Computer Games, and Software Studies (Cambridge, MA: MIT Press, 2009); computer forensics, Matthew G. Kirschenbaum, Mechanisms: New Media and the Forensic Imagination (Cambridge, MA: MIT Press, 2008); media archaeology, Jussi Parikka, What is Media Archaeology? (Cambridge, MA: Polity, 2012), and Media Archaeology: Approaches, Applications, and Implications, ed. Erkki Huhtamo and Jussi Parikka (Berkeley: University of California Press, 2012); and critical code studies, Nick Montfort et al., 10 Print Chr \$205.5+RND(1)); : GOTO 10 (Cambridge, MA: MIT Press, 2013). These disciplines are also not mutually exclusive, and much 


\section{The Last of Us and The Last of Us Remastered 463}

born-digital text and electronic record to trace its transmission through different material forms or cite a specific digital artifact so others can identify the version under analysis are not of primary concern to these disciplines. The Game Metadata and Citation Project (GAMECIP), a multi-year Institute of Museum and Library Services-funded investigation of metadata needs and citation practices surrounding computer games in institutional collections, recently published two reports outlining a metadata scheme for cataloguing videogames and a best-practices approach to cataloguing videogames using RDA and MACR 21 metadata standards. ${ }^{9}$ While these reports are an important contribution to the bibliographic description of videogames, they do not, as of writing this paper, provide any guidelines for the bibliographical citation of videogames. Nathan Altice in his platform studies book I am Error: The Nintendo Family Computer / Entertainment System Platform, does provide an appendix for how to cite videogame cartridges and emulated copies, but acknowledges that his approach is limited to platform-specific knowledge of the Nintendo Entertainment System. ${ }^{10}$ Ryan Cordell recently presented a paper at the Bibliography and Scholarly Editing Forum's panel at the Modern Languages Association 2016 conference, outlining a case-study approach for how we may describe and cite digital artifacts, such as the electronic records of newspapers, and their "dirty OCR" editing procedures. ${ }^{11}$ Cordell, like Altice and the GAMECIP project, have made important contributions to the description and citation of born-digital texts and electronic records, but there is

of the literature overlaps theoretically and methodologically in their analysis of materials forms and texts.

9. Greta de Groat et al., "Core Metadata Schema for Cataloguing Video Games Version 1: Game Metadata and Citation Porject (GAMECIP) Tech Report 1" (GAMECIP, December 2015), and Online Audiovisual Catalogers, Inc., Cataloging Policy Committee Video Game RDA Best Practices Task Force, "Best Practices for Cataloging Video Games: Using RDA and MARC21" (Online Audiovisual Catalogers, Inc., June 2015), http:// https://gamecip.soe.ucsc.edu/publications.

10. Nathan Altice, I am Error: The Nintendo Family Computer / Entertainment System Platform (Cambridge, MA: MIT Press, 2014), 333-41.

11. Cordell's analysis of the Chronicling America database of digitized texts demonstrates how the ubiquity of born-digital texts and electronic records in humanities scholarship has offset our considerations for the accuracy and authenticity of the OCR surrogate. Ryan Cordell, “Q $\mathrm{Q}$-jtb the Raven': Taking Dirty OCR Seriously," Ryan Cordell, 7 January 2016, accessed 25 February 2016, http://ryancordell .org/research/qijtb-the-raven/. 


\section{$464 \quad$ Bibliographical Society of America}

still much to be done. As Altice noted, current "citations adhere to the barest enumerative models", failing to include basic information, such as platform and material format release, file format, and file version. ${ }^{12}$ Without such detailed descriptions, how will we know which edition, or issue of that edition, scholars are playing and analyzing in their research of videogame texts?

Adopting Matthew Kirschenbaum's terms for the description of born-digital texts and electronic records-layer, version, release, object, state, instance, and copy-I present a case-study of The Last of $U s$ and The Last of Us Remastered that reveals some of the secretive publishing and manufacturing practices of the videogame industry, and suggest a potential way forward for the description and citation of $\mathrm{PS}_{3}$ and $\mathrm{PS}_{4}$ videogames released on Blu-ray discs and digital downloads through the PS Store. ${ }^{13}$ I only discuss the platform-specific knowledge of Sony Computer Entertainment and their $\mathrm{PS}_{3}$ and $\mathrm{PS}_{4}$ consoles and videogames, specifically the editions of The Last of $U_{s}$ and The Last of $U_{s}$ Remastered. However, in doing so I provide a close reading of The Last of $U s$ texts that furthers our knowledge and discussion of the bibliography of videogames. Alan Galey performed a similar close reading in his case study of the variant paper and electronic versions of the novel The Sentimentalist, revealing numerous format-specific variants of The Sentimentalist texts as they were transmitted through different material forms. ${ }^{14}$ Such an approach draws out the minutiae critical to developing a field of research around the bibliography of videogames that may not be as perceptible in a much larger macro analysis of born-digital texts and electronic records. However, such macro-analysis studies, or distantreading projects, will be essential in the future if the field of bibliography is to develop systems of documentation for the bibliography of videogames and other born-digital texts and electronic records.

\section{II}

Videogames, much like books, are released as editions. Many videogames, when they are first released, do not provide edition documentation on any physical or digital aspects of the distributed format. These

12. Altice, I am Error, 334 .

13. See Matthew Kirschenbaum, "Editing the Interface: Textual Studies and First Generation Electronic Objects," Text 14 (2002): 43-51.

14. Alan Galey, "The Enkindling Reciter: E-Books in the Bibliographical Imagination," Book History 15 (2012): 210-47. 


\section{The Last of Us and The Last of Us Remastered 465}

first editions of videogames are colloquially referred to as standard editions because they are typically released without any additional paratexts, such as physical and digital objects and texts. ${ }^{15}$ When subsequent versions are released with these additional paratexts, they are frequently labelled as an edition to distinguish them from the standard edition. Currently, there are fourteen publically released authorized editions for The Last of $U s$ for the $\mathrm{PS}_{3}$ and The Last of Us Remastered on the $\mathrm{PS}_{4}{ }^{16}$ These authorized editions are published by SCEA and licensed to Sony Computer Entertainment's other videogame publishers for distribution: Sony Computer Entertainment Incorporated (SCEI), Sony Computer Entertainment Europe (SCEE), and Sony Computer Entertainment Worldwide Studios (SCE WWS). ${ }^{17}$ Table 1 lists the current authorized editions of the two titles, noting their regional distributors. ${ }^{18}$

These regional distributors roughly correspond to four geographical regions: North and South America, Asia, Europe, and Oceania. However, the regional publishing practices are far more complicated. The

15. The paratexts associated with the release of a videogame can vary with the type of edition. Most of these paratexts include digital add-ons, such as DLC that can include avatars, standalone games, additional levels, and other digital texts. Sometimes the physical release of a videogame can include material objects and texts, such as figurines, comics, and supporting documentation.

16. In the case of The Last of $U s$, an authorized edition is a videogame that has been manufactured and distributed by a licensed organization of Sony Computer Entertainment, which can include Sony Computer Entertainment Incorporated, Sony Computer Entertainment Europe, Sony Computer Entertainment America, and Sony Computer Entertainment Worldwide Studios. While press release and tester copies of videogames can also be considered authorized editions by Sony Computer Entertainment, they are not released publically and commercially and therefore fall outside the scope of what would be considered an authorized release for commercial mass distribution.

17. Sony Computer Entertainment recently announced they would also be restructuring their organization, merging Sony Computer Entertainment and Sony Network Entertainment into Sony Interactive Entertainment (SIE) to integrate their hardware, software, content, and network operations, headquartered in San Mateo, California. It is unclear how this restructuring will affect future videogame releases, including potential re-releases of The Last of Us trademark (Sony Computer Entertainment, "Sony Computer Entertainment and Sony Network Entertainment Announce the Formation of Sony Interactive Entertainment LLC, Sony Computer Entertainment Inc., 26 January 2016, accessed 25 February 2016, http:// www.scei.co.jp/en/corporate/release/2016/160126a.html).

18. It is unclear whether this table is comprehensive for the authorized editions licensed by Sony Computer Entertainment of America for commercial release. 
TABLE 1

List of authorized editions for The Last of $U$ s and The Last of Us Remastered.

\begin{tabular}{lll} 
Edition & Format Release & Regional Distributors \\
\hline Standard & BR/DD & SCEA, SCEI, SCEE, SCE WWS \\
Ellie edition & BR/DD & SCEE \\
Joel edition & BR & SCEE \\
Ellie Collector's edition & BR & SCEE (Italy) \\
Joel Collector's edition & BR & SCEE (Italy) \\
Post-Pandemic edition & BR & SCEA \\
Survival edition & BR & SCEA \\
Collector's edition & BR & SCEI \\
Special edition & BR & SCEI \\
Steelbook edition & BR & SCEI \\
Game of the Year (GOTY) & BR/DD & SCEE, SCEI \\
The Best & BR/DD & SCEI (Japan) \\
Standard Remastered & BR/DD & SCEA, SCEI, SCEE, SCE WWS \\
Remastered Steelbook & BR & SCEE (Germany)
\end{tabular}

SCEE, while publishing videogames for European markets, also publish games in some African and Asian countries, such as South Africa, Kuwait, and Saudi Arabia, to name a few. Further, SCEE publishes all the PlayStation videogames in Oceania, such as Australia and New Zealand, even though the region code for Oceania is different from the European region. ${ }^{19}$ In Asia, excluding Japan and South Korea, most of the physical releases of $\mathrm{PS}_{3}$ and $\mathrm{PS}_{4}$ games are distributed by SCEI,

19. There are currently four region codes for PlayStation games that broadly represent the following continents and countries: Region 1, North and South America; Region 2, Europe, the Middle East, South Africa, and Japan; Region 3, Asia excluding Japan; Region 4, Australia and New Zealand. These region codes were first used on the PlayStation and PlayStation 2 to prevent console owners from playing videogames sold in foreign markets, such as Japanese games being played on American consoles. With the release of the $\mathrm{PS}_{3}$, these regional lockouts were removed, and now players can play any $\mathrm{PS}_{3}$ or $\mathrm{PS}_{4}$ game on their respective console. That being said, if a $\mathrm{PS}_{3}$ or $\mathrm{PS}_{4}$ game includes specific regional codes allowing access to exclusive content, those codes will only work for a PlayStation Network account associated with that region. For example, physical copies of The Last of $U_{s}$ include online-pass vouchers. If I were to play a Japanese copy of the videogame on an American console, I would need a Japanese PlayStation Network account to use that voucher. 


\section{The Last of Us and The Last of Us Remastered 467}

whereas the digital download releases are distributed by SCE WWS via the PS Store.

Sony Computer Entertainment's publishing system gets even more complicated once we observe the physical and digital download releases of The Last of Us and The Last of Us Remastered on the $\mathrm{PS}_{3}$ and $\mathrm{PS}_{4}$. Table 2 lists the current product codes for The Last of Us trademark released on the $\mathrm{PS}_{3}$ and $\mathrm{PS}_{4}$, organized by console, region code, publisher, and distribution format.

Currently, there are twenty-seven product codes for The Last of $U s$ trademark that represent the variant videogame editions released since

TABLE 2

List of product codes for The Last of $U s$ and

The Last of Us Remastered (see figure 1).

\begin{tabular}{|c|c|c|c|c|c|}
\hline Console & Region & Publisher & Format & Code & Edition \\
\hline $\mathrm{PS}_{3}$ & 1 & SCEA & DD & NPUA-8096o & Standard (Americas) \\
\hline $\mathrm{PS}_{3}$ & 1 & SCEA & $\mathrm{BR}$ & BCUS-98174 & Standard (Americas) \\
\hline $\mathrm{PS}_{3}$ & 2 & SCEE & $\mathrm{BR} / \mathrm{DD}$ & BCES-01585 & GOTY (Europe) \\
\hline $\mathrm{PS}_{3}$ & 2 & SCEE & $\mathrm{BR} / \mathrm{DD}$ & BCES-o1584 & Standard (Europe) \\
\hline $\mathrm{PS}_{3}$ & 3 & SCE WWS & $\mathrm{DD}$ & NPHA-80243 & GOTY (Asia) \\
\hline $\mathrm{PS}_{3}$ & 3 & SCE WWS & DD & NPHA-80243 & Standard (Asia) \\
\hline $\mathrm{PS}_{3}$ & 3 & SCEI & BR & BCAS-25024 & GOTY (Asia) \\
\hline $\mathrm{PS}_{3}$ & 3 & SCEI & $\mathrm{BR}$ & BCKS-15018 & GOTY (Korea only) \\
\hline $\mathrm{PS}_{3}$ & $\begin{array}{l}3 \\
3\end{array}$ & SCEI & $\mathrm{BR}$ & BCAS-25015 & Standard (Asia) \\
\hline $\mathrm{PS}_{3}$ & 3 & SCEI & $\mathrm{BR}$ & BCAS-20270 & Standard (Asia)+DLC \\
\hline $\mathrm{PS}_{3}$ & 3 & SCEI & DD & NPJA-ooog6 & Standard (Japan only) \\
\hline $\mathrm{PS}_{3}$ & 3 & SCEI & $\mathrm{BR}$ & BCJS-37010 & Standard (Japan only) \\
\hline $\mathrm{PS}_{3}$ & 3 & SCEI & $\mathrm{BR}$ & BCKS-15013 & Standard (Korea only) \\
\hline $\mathrm{PS}_{3}$ & 3 & SCEI & DD & NPJA-ooog6 & The Best (Japan only) \\
\hline $\mathrm{PS}_{3}$ & 3 & SCEI & $\mathrm{BR}$ & BCJS-75004 & The Best (Japan only) \\
\hline $\mathrm{PS}_{3}$ & 4 & SCEE & $\mathrm{BR} / \mathrm{DD}$ & BCES-01585 & GOTY (Europe) \\
\hline $\mathrm{PS}_{3}$ & 4 & SCEE & $\mathrm{DD}$ & NPEA-00435 & Standard (Europe) \\
\hline $\mathrm{PS}_{3}$ & 4 & SCEE & $\mathrm{BR}$ & BCES-01584 & Standard (Europe) \\
\hline $\mathrm{PS}_{4}$ & 1 & SCEA & $\mathrm{BR} / \mathrm{DD}$ & CUSA-oo $55^{2}$ & Standard (Americas) \\
\hline $\mathrm{PS}_{4}$ & 2 & SCEE & $\mathrm{BR} / \mathrm{DD}$ & CUSA-00557 & Standard (Eng/Pol/Rus) \\
\hline $\mathrm{PS}_{4}$ & 2 & SCEE & $\mathrm{BR} / \mathrm{DD}$ & CUSA-00556 & Standard (Europe) \\
\hline $\mathrm{PS}_{4}$ & 3 & SCE WWS & $\mathrm{DD}$ & CUSA-00559 & Standard (Eng/Chinese/Korean) \\
\hline $\mathrm{PS}_{4}$ & 3 & SCEI & BR & PCAS-02004 & Standard (English/Chinese) \\
\hline $\mathrm{PS}_{4}$ & 3 & SCEI & BR & PCJS-53003 & Standard (Japan only) \\
\hline $\mathrm{PS}_{4}$ & 3 & SCEI & DD & CUSA-00554 & Standard (Japan only) \\
\hline $\mathrm{PS}_{4}$ & 3 & SCEI & $\mathrm{BR}$ & PCKS-92003 & Standard (Korea only) \\
\hline $\mathrm{PS}_{4}$ & 4 & SCEE & $\mathrm{BR} / \mathrm{DD}$ & CUSA-0o556 & Standard (Europe) \\
\hline
\end{tabular}




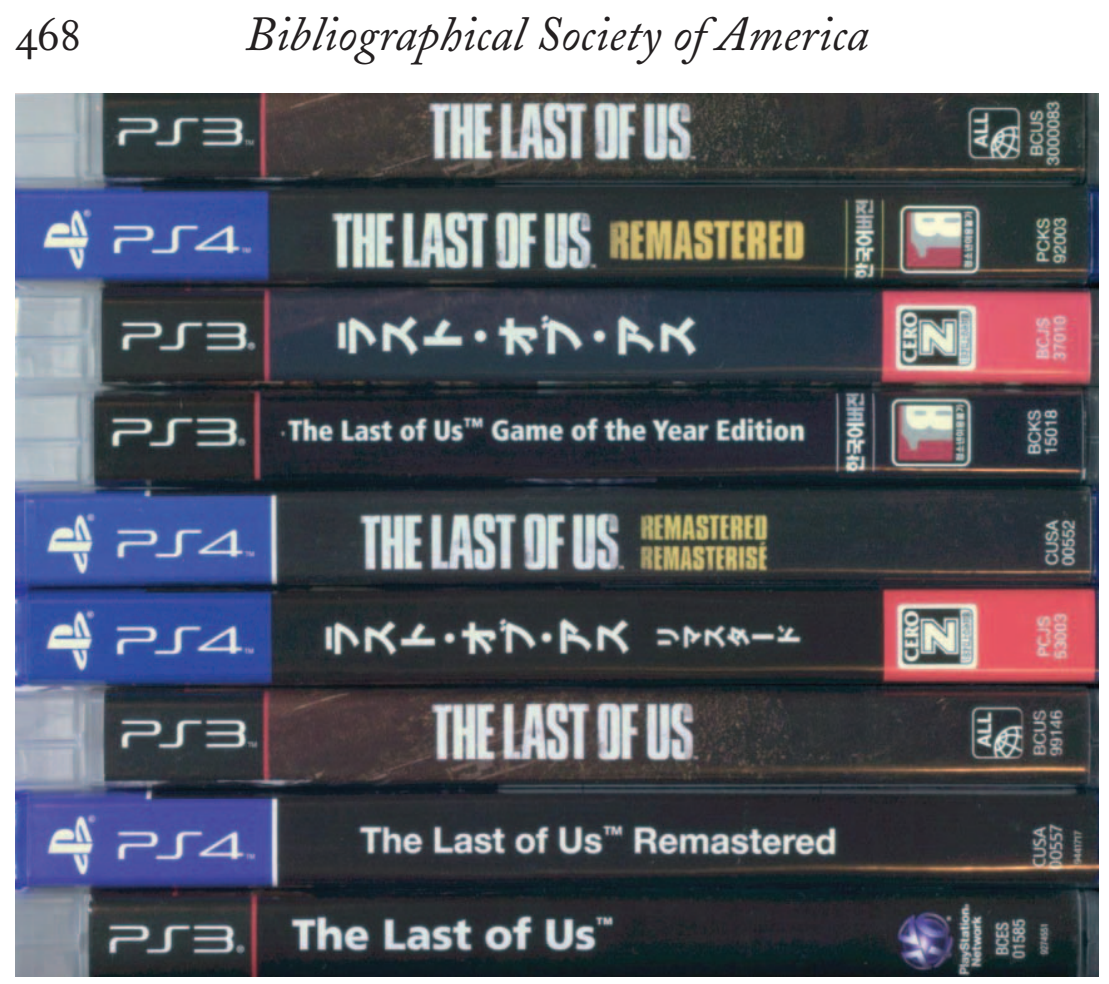

Fig. 1: Image of several copies of The Last of Us and The Last of Us Remastered released by the publishers of Sony Computer Entertainment. Photo by author.

June 2013 by Sony Computer Entertainment's distributors. ${ }^{20}$ These product codes, though distributed as Blu-ray discs and digital downloads by different publishers, can be categorized into three substantial releases of The Last of $U s$ trademark: the standard edition first released 14 June 2013 for the PS 3 ; the Game Of The Year (GOTY) edition first released 12 November 2014 for the $\mathrm{PS}_{3}$ that includes the original game plus the

20. This table may not be exhaustive. While I am confident I have found all of the authorized product codes for The Last of Us trademark, there may be more. Japan and South Korea stand out as unique examples with their own product codes, and there may be more unique product codes falling under similar criteria to Japan and South Korea's special publishing status in Sony Computer Entertainment. There are, of course, numerous local product numbers that can be found on the packaging of the numerous editions, but the product codes for $\mathrm{PS}_{3}$ and $\mathrm{PS}_{4}$ releases for The Last of $U$ s trademark can be found on the physical Blu-ray disc or in the URL of the PS Store. 


\section{The Last of Us and The Last of Us Remastered 469}

majority of the DLC released since 14 June 2013; and the standard $R e$ mastered edition first released for the $\mathrm{PS}_{4}$ on 29 July 2014.

However, just because a videogame is labelled an edition or is released on a different date by a publisher and distributor does not mean it represents a new edition in the bibliographical sense of the term. Thomas Tanselle proposed a definition that fits quite neatly with the format of videogames:

An edition should be defined as all copies resulting from a single job of typographical composition. Thus, whether printed from type (set by hand or by machine), or plates, or by means of photographic or electronic process, all copies that derive from the same initial act of assembling the letterforms belong to the same edition. ${ }^{21}$

As all videogames are born-digital typographical compositions of text, (or code as is more frequently used in videogame development), they tidily fit into Tanselle's definition of edition as an electronic process. Further, his statement, "all copies that derive from the same initial act of assembling the letterforms belong to the same edition," unambiguously defines an edition based on the total assemblage of text, not the publication decisions of a publisher. As noted earlier, there were three substantial releases of The Last of Us represented by fourteen authorized editions and twenty-seven product codes, but do these releases represent unique editions in the bibliographical sense, or are they just variant issues and states of The Last of Us and The Last of Us Remastered? While there are various physical and digital paratexts that accompany the standard edition for The Last of $U_{s}$ on the $\mathrm{PS}_{3}$, it is important to note that the product codes for all of the authorized editions were released with the same printed product code on the Blu-ray discs. For example, the Pandemic and Survival editions distributed by the SCEA as physical Blu-ray discs included the same product code as the standard edition. ${ }^{22}$ This is also the case for the authorized editions distributed as physical releases by the SCEI and SCEE simultaneously to their respective standard editions. Moreover, the GOTY editions, distributed only by SCEE, SCEI, and SCE WWS, simultaneously replaced their standard edition precursors upon their November 2014 release through the PS

21. Thomas Tanselle, "The Bibliographical Concepts of Issue and State," The Papers of the Bibliographical Society of America 69 (1975): 18.

22. The product code for those two editions is BCUS-98174. 
Store, indicating that the compiled code for The Last of Us standard editions is the same as the GOTY editions. This observation is further supported by the bibliographical evidence that the standard edition product code distributed by the SCEI in Asia is the same as the GOTY edition. ${ }^{23}$ However, the GOTY edition still represents a significant release of The Last of $U s$ because it replaced the standard edition on SCEE and SCE WWS PS Stores, and, more importantly, is the only $\mathrm{PS}_{3}$ edition of The Last of $U s$ available on those PS Stores.

Further evidence appears in the patch notes and software update descriptions for The Last of $U_{s}$ and The Last of $U_{s}$ Remastered. They are categorically separated into version updates for either the $\mathrm{PS}_{3}$ or $\mathrm{PS}_{4}$, not for any particular edition released by a Sony Computer Entertainment distributor. ${ }^{24}$ Moreover, the software updates for the $\mathrm{PS}_{3}$ and $\mathrm{PS}_{4}$ are universal across all of The Last of $U s$ and The Last of $U s$ Remastered editions, ranging from 1.1 to 1.11 on the $\mathrm{PS}_{3}$ and 1.1 to 1.06 on the $\mathrm{PS}_{4}$. The universal application of the software updates for either the $\mathrm{PS}_{3}$ or $\mathrm{PS}_{4}$ suggests that every copy of the $\mathrm{PS}_{3}$ release of the The Last of $U$, including all the authorized and GOTY editions distributed by the SCEA, SCEE, SCEI, and SCE WWS, uses the same executable files compiled from the same assemblage of code. This is a critical feature of these texts, because versions of executable files can only be updated by software patches running on the same code. This fundamental process of all software is particularly apparent when one considers the version control of word processing software to create born-digital texts. As you use the save feature for the text file you have created, the version of your file changes. Further, you can only make those changes to the document you are editing. Videogame development follows a similar control protocol, where any changes made to a videogame are pushed as version updates in the form of software patches. For one software patch to update all authorized editions of The Last of Us on the $\mathrm{PS}_{3}$ or The Last of $U s$ Remastered on the $\mathrm{PS}_{4}$, those editions all need to be copies of the same assemblage of code, the same compiled executable file. Following this essential aspect of version control for any digital text, there can only be two executable files for The Last of $U s$ according to Naughty Dog

23. The standard edition and the GOTY edition distributed by the SCEI is NPHA-80243.

24. Descriptions of patch notes and software updates can be found posted on the Naughty Dog Studios blog, accessed 25 February 2016, http://www.naughtydog .com/blog/. 
Studios patch and update notes: The Last of $U_{s}$ released on the $\mathrm{PS}_{3}$, and The Last of Us Remastered released on the $\mathrm{PS}_{4}$. Despite fourteen authorized editions and twenty-seven product codes of The Last of $U s$ and The Last of Us Remastered distributed by SCEA, there are only two editions of The Last of Us trademark in the bibliographical sense.

\section{III}

Now that I have established there are only two editions of The Last of $U$ s trademark, I will go through each level of bibliographic description using Kirschenbaum's concepts of layer, version, release, object, state, and instance to frame my analysis, concluding with a proposed citation for The Last of $U_{s}$ and The Last of Us Remastered released on Blu-ray discs and as digital downloads via the PS Store. As Kirschenbaum argued, the traditional bibliographical terminology of edition, impression, and state, as outlined by Fredson Bowers in Principles of Bibliographical Description, are:

absolutely dependent on the material specificity of their medium - that is their great value. The disjunctions between the material conditions of printed and electronic objects are, I believe, too great to bridge. . . . It seemed more effective to introduce a new set of terms, conceived expressly for the electronic medium..$^{25}$

While many of the bibliographical definitions developed by scholars over the years may not necessarily be adequate for the description of born-digital texts and electronic records, the concepts underlying them are invaluable guides to understanding the computational definitions of layer, version, release, object, state, instance, and copy. It is no coincidence that videogames are released and labelled as an edition by publishers. Videogames share as much heritage with book publishing practices as they do with the history of software development. Acknowledging this shared heritage allows for the digital minutiae of videogames to be accessed and described as published born-digital texts and systems of computational expression.

Kirschenbaum defines layer as "a building block in models of computer networking, where it represents a discrete communication protocol," that is, "all elements of an electronic work that are both computationally compatible and functionally integrated." In the videogame sense of this concept, layer can refer to console platforms, such as the $\mathrm{PS}_{3}$ and $\mathrm{PS}_{4}$,

25. Kirschenbaum, "Editing the Interface," 46. 
but can also include game engines, web browsers, software platforms, or other discrete communication protocols that enable the transmission of executable files at any stage of development. Furthermore, "when one or more elements are ported such that the whole is no longer computationally compatible or functionally integrated, then a new layer is created." ${ }^{26}$ In the context of The Last of $U s$, it is evident that there are two layers: the $\mathrm{PS}_{3}$ and $\mathrm{PS}_{4}$. Kirschenbaum's concept of layer is similar to Tanselle's definition of edition in the bibliographical sense that electronic texts are part of the same work if they are derived from the same assemblage of code. Indeed, where their definitions overlap is in their statement that a text is part of the same edition or layer if the text is from "a single job of typographical composition" or is "computationally compatible and functionally integrated." ${ }^{27}$ But there are also important areas where the two concepts do not overlap. Whereas an edition can be determined through forensic analysis of the material object, a layer is determined either through access to mediated computational environments or an analysis of paratextual evidence, such as patch notes. Due to the nature of videogame consoles, where data is hidden behind copyright, intellectual property, and trademark protections, and locked by digital rights management (DRM) tools and mechanisms, it is both technically and legally difficult to look "under-the-hood" of a platform or videogame's code. Hacking these DRM's, though conducted for the purposes of research under the terms of fair use, is potentially susceptible to legal prosecution. For the purposes of this paper though, forensic analysis has been limited to distinctly observable phenomena found on openly accessible information on the hardware, software, and paratextual documents of The Last of Us editions for the $\mathrm{PS}_{3}$ and $\mathrm{PS}_{4}$ platform environments. While it cannot be computationally proven that all copies of The Last of $U_{s}$ run on the same $\mathrm{PS}_{3}$ layer and all copies of The Last of Us Remastered run the same $\mathrm{PS}_{4}$ layer, there are enough file metadata and paratextual documents to suggest that this is in fact the case. ${ }^{28}$

26. Ibid.

27. Tanselle, "The Bibliographical Concepts of Issue and State," 18; Kirschenbaum, "Editing the Interface," 46.

28. To briefly test this hypothesis, I installed multiple copies of The Last of Us and The Last of Us Remastered onto several $\mathrm{PS}_{3}$ and $\mathrm{PS}_{4}$ consoles. These copies had different product codes and all were successfully installed onto the consoles. While I did not have physical access to all product codes, which would have been a 


\section{The Last of $U$ s and The Last of Us Remastered 473}

Below the layer of an electronic work is its version and release. Kirschenbaum defines version as "cumulative iterations of code." When a software patch is released for a videogame, the executable file is computationally altered to functionally integrate in a layer as a new version of the videogame. Developers will release patches to fix bugs and errors but also to update out-dated content with new DLC. Kirschenbaum employs the version number of files to distinguish one version from another, such as how The Last of $U_{s}$ on the $\mathrm{PS}_{3}$ can vary from version 1.0 to $1.11 .^{29}$ Figure 2 shows an example of a version number for The Last of $U s$ on the $\mathrm{PS}_{4}$ platform environment. Alterations to The Last of $U s$ usually include bug fixes to software errors and the tweaking of weapon perks and abilities based on player feedback from The Last of Us community. The latter of these computational alterations would affect important player aspects, such as gameplay. For example, the software patches 1.11 for the $\mathrm{PS}_{3}$ and 1.06 for the $\mathrm{PS}_{4}$ included new DLC, such as character gestures, survival skills, and weapons for the Factions multiplayer feature..$^{30} \mathrm{Up}$ until this update, players of any of the previous versions would not have had access to these new gameplay features. As a result, this new version of The Last of $U$ s editions is arguably not only a new version but also a variant videogame of the previous version. It is critical, therefore, that such variants are taken into consideration when citing and describing the play experience of a videogame.

Following the version of a videogame is its release. According to Kirschenbaum, "different releases of the work are all computationally compatible with one another, but they are not functionally integrated." ${ }^{11}$

financially expensive endeavour, I was able to use at least one regional code released by each of the Sony Computer Entertainment publishers to verify that at least the copies I tested functioned across $\mathrm{PS}_{3}$ and $\mathrm{PS}_{4}$ consoles.

29. Accessing the version number on any $\mathrm{PS}_{3}$ or $\mathrm{PS}_{4}$ console is relatively straightforward. Before opening The Last of $U s$ or The Last of Us Remastered application on the $\mathrm{PS}_{3}$ or $\mathrm{PS}_{4}$ home screen, anyone can press either the " $\square$ " or "Options" button to view the current version of the software. While developers do not always provide detailed patch notes and update descriptions with the update on the $\mathrm{PS}_{3}$ or $\mathrm{PS}_{4}$ consoles, more detailed information can usually be located on the studios' website.

30. ermonacelli, "The Last of Us Patch 1.11 and 1.06 - New MP Add-Ons," Naughty Dog Studios, 9 December 2014, accessed 25 February 2016, http://www .naughtydog.com/site/post/the_last_of_us_patch_111_and_106_new_mp_add_ons/.

31. Kirschenbaum, "Editing the Interface," 47. 


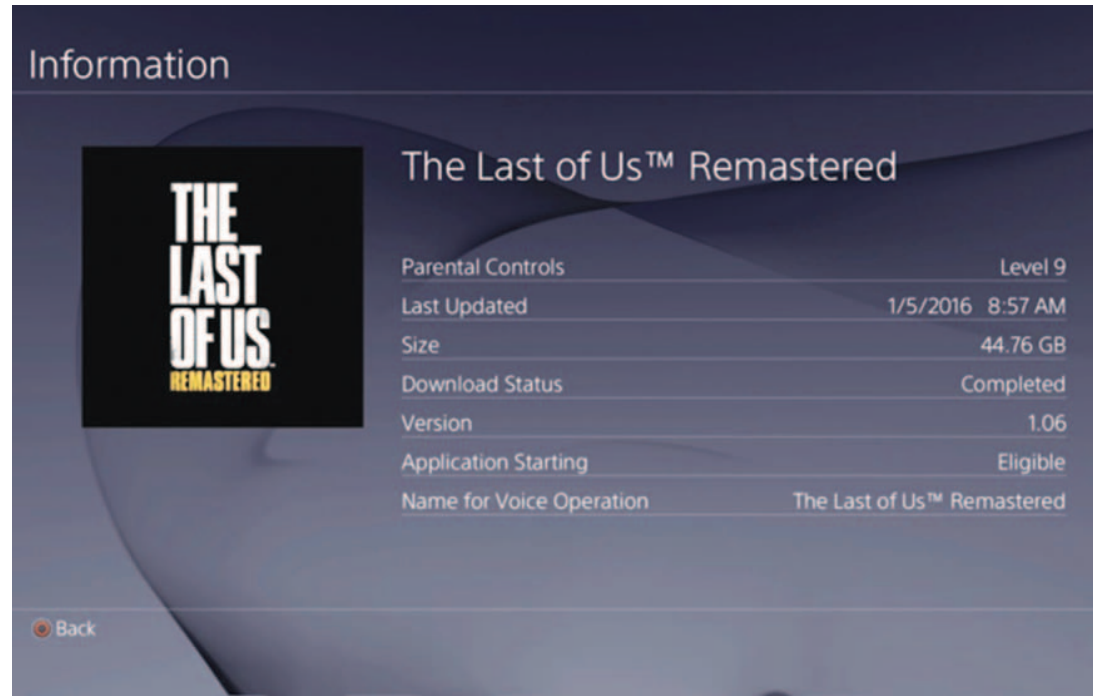

Fig. 2: Example of a version number for The Last of Us Remastered on the $\mathrm{PS}_{4}$ software environment. Screenshot by author.

As noted earlier, all copies of The Last of Us and The Last of Us Remastered are computationally compatible with each other on the respective consoles of the $\mathrm{PS}_{3}$ and $\mathrm{PS}_{4}$, but this does not mean they are functionally integrated in the computational sense that they run on the same physical or digital release, such as a Blu-ray Disc or a digital download via the PS Store. ${ }^{32}$ In the case of The Last of $U s$, there are three elements that identify a release: the product codes, the product identifier, and the issue number. The product codes for The Last of $U_{s}$ and The Last of $U_{s}$ Remastered represent the different releases of The Last of $U s$ trademark that are not functionally integrated with one another. Figure 3 shows where the product codes are located on the front of the Blu-ray disc, while figure 4 shows where the product codes are located in the PS Store URL. Though this code may be located on other packaging, such

32. When I installed multiple copies of The Last of Us and The Last of Us Remastered that were released by different publishers with different product codes onto the $\mathrm{PS}_{3}$ and $\mathrm{PS}_{4}$, it created new applications for the respective consoles. This meant that while the videogame releases were computationally compatible, they were not functionally integrated. Only videogames with the same product codes were functionally integrated. 

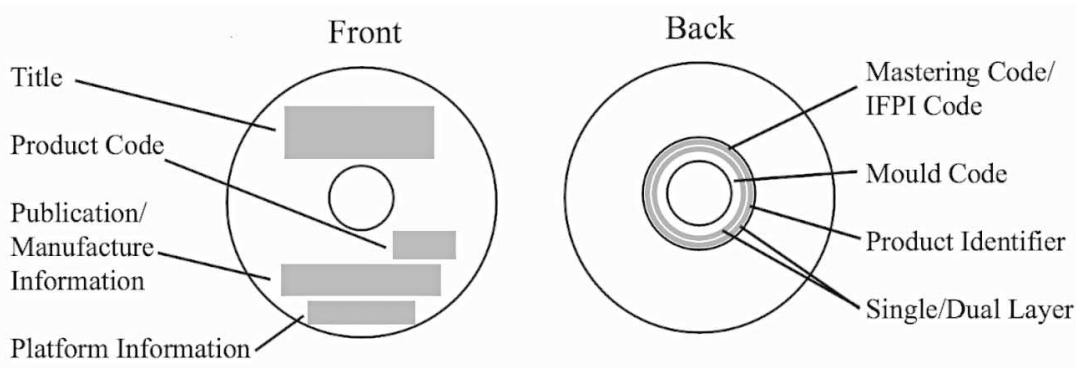

Fig. 3: The anatomy of the front and back of a Blu-ray disc for the $\mathrm{PS}_{3}$ and $\mathrm{PS}_{4}$. Graphic by author.

as the disc case, the primary location for this piece of information is on the Blu-ray disc itself. The location of these product codes will also vary slightly from release to release depending on the distributor's house style, but it will always be located on the front of the Blu-ray disc for $\mathrm{PS}_{3}$ or $\mathrm{PS}_{4}$ titles.

Product identifiers are located on the back of Blu-ray discs and in the PS Store URL, as shown in figure 4. Occasionally the product identifier on the back of the Blu-ray disc is the same as the product code. Titles published by the SCEA include different product codes and product identifiers, such as BCUS-98174 and BPDL-453050 for The Last of Us on the $\mathrm{PS}_{3}$, whereas the SCEI and SCEE Blu-ray discs do not. These product identifiers are also occasionally printed on the front of the Bluray disc alongside the product code, as is the case with The Last of $U$ s and The Last of Us Remastered distributed by the SCEA. However, another code that can be used in addition to the product identifier for Blu-ray discs are the Source Identification (SID) codes established by the International Federation of the Phonographic Industry (IFPI). SID codes are printed on optical discs, such as Blu-ray discs, to identify the manufacturer, along with equipment and master discs used to create each disc, for the purposes of combating copyright infringement. Figure 3 also shows where SID codes can be located, including the SID mastering code, which identifies the manufacturing facility used to create a master from which moulds are produced, and the SID mould code, which identifies the plant where the disc was pressed. In many cases, these two SID codes use the same IFPI number because the manufacturing facility and the plant are located together; this is the case with The Last 


\section{Bibliographical Society of America}

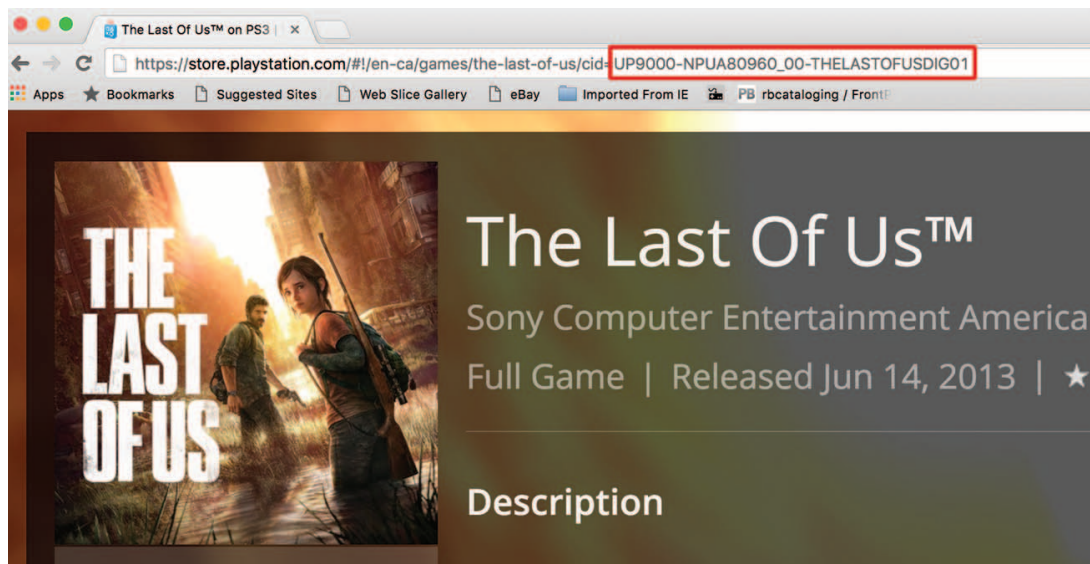

Fig. 4: Example of the The Last of Us digital download on the PS Store. Screenshot by author.

of $U s$ distributed by the SCEA, manufactured in Terre Haute, Indiana, with the SID code IFPI L322. There are also instances where two different numbers are provided, as is the case with The Last of Us Remastered distributed by the SCEI for the Japanese market, manufactured in Shizuoka-Haibara District, Shizuoka Prefecture, with the SID codes IFPI L279 and IFPI L280. However, for all the authorized Blu-ray disc copies of The Last of Us, there will always be two codes provided, even if they are the same SID code, because their Blu-ray discs are dual layered to contain ${ }_{5} \mathrm{OGB}$ of data. When a disc is a single-layer, it contains ${ }_{25} \mathrm{~GB}$ of data and only provides one IFPI code on the ring layer, though it may provide an SID mould code further to the interior of the disc, as shown in figure $3 .{ }^{33}$ The file size for The Last of $U_{s}$ and The Last of $U_{s}$ Remastered releases varies between $25-30 \mathrm{~GB}$ and $35-45 \mathrm{~GB}$, respectively, not only because of the improved aesthetics in the Remastered edition, but also because of the number of languages and subtitle supports for

33. It should be noted here that none of The Last of Us and The Last of Us Remastered editions includes an SID mould code on the interior of the Blu-ray disc. Instead, they include the PlayStation logo along with the $\mathrm{PS}_{3}$ and $\mathrm{PS}_{4}$ logos, respectively. As the SID mould code indicates the plant where the Blu-ray disc was pressed, these logos would suggest that these discs were manufactured within the $\mathrm{PS}_{3}$ or $\mathrm{PS}_{4}$ plant or division, within their respective Sony facilities, and thus maybe not requiring a unique SID mould number. 


\section{The Last of Us and The Last of Us Remastered}

releases distributed by the different Sony Computer Entertainment publishers to accommodate their respective linguistic markets. As such, they are all manufactured as dual-layer Blu-ray discs and include at least two SID codes, one for each layer. Much like the printer and publisher information located in the title-page imprint or the colophon found at the end of a book, manufacturer information on the pressing of a videogame is embedded in minuscule text on the back of the optimal disc. Ironically, this cryptic information is only provided to ensure authenticity of the $\mathrm{PS}_{3}$ or $\mathrm{PS}_{4}$ product, not to acknowledge the manufacturers themselves. ${ }^{34}$

The issue number is the third and final piece of bibliographical information relevant to the release of The Last of $U s$ trademark. The issue number represents the specific country a videogame is released in, whether as a Blu-ray disc by one of the sixty-three national distributors or on one of the ninety-three PS Stores. ${ }^{35}$ While the issue number for Blu-ray discs is provided on disc covers as paratextual information, much like ISBNs in books, where the disc is distributed can affect how it is "functionally integrated" into the platform environment. As noted earlier, one of the paratexts for The Last of $U_{s}$ for the $\mathrm{PS}_{3}$ is an onlinepass voucher that was also included with the standard edition and the other editions distributed by the SCEA. These vouchers are coded specifically for the PS Store in which they are nationally distributed, meaning that if you buy a Japanese release of The Last of Us and want to

34. Sony Computer Entertainment does not willingly provide this manufacturing information. Much of this information has been collected and quantified in wikis for developers to understand the manufacturing process of their videogames if they reach international markets ("Disc Identification/Serialization Data," $P S_{4}$ Developer Wiki, 10 July 2015, accessed 25 February 2016).

35. New national distributors and PS Stores continue to open, and so their number may have increased since writing this paper. In 2015, for example, PS Stores opened in Guatemala, El Salvador, Honduras, and Paraguay; both The Last of Us and The Last of Us Remastered were released on 11 June 2015 in those PS Stores. Moreover, there are sometimes several PS Stores in one country to accommodate multilingual populations, such as the Canadian English and French PS Stores. It should also be noted that not all distributing countries have PS Stores. For example, Vietnam releases PS $_{3}$ consoles and videogame discs but does not have a PS Store. As well, it is important to keep in mind that not all national distributors and PS Stores may release all PlayStation products due to marketing and censorship practices within those countries. 


\section{$478 \quad$ Bibliographical Society of America}

access its networked features, you will need to have a Japanese PS Store account. So while the Japanese Blu-ray disc will play on an American console, the American user will not be able to access its networked features. The same restriction applies to PS Store digital downloads of The Last of $U_{s}$ titles, albeit with more hindering effects. If you purchase The Last of $U_{s}$ or The Last of Us Remastered on the $\mathrm{PS}_{3}$ or $\mathrm{PS}_{4}$, the entire copy of that videogame will only work with the PS Store from which it was purchased. ${ }^{36}$ However, as PS accounts can be linked with more than one console, a player can travel with his or her account and reinstall the game on another console in another geographic location. So while issue numbers may not be directly located on the physical digital format of the videogame, the paratextual information is important for contextualizing how the videogame is functionally integrated on a computational level within the $\mathrm{PS}_{3}$ or $\mathrm{PS}_{4}$ consoles.

While Kirschenbaum's term release has been useful in framing how videogame editions can be computationally compatible but not functionally integrated, it is limited in its scope to distinguishing different aspects of a release. When explaining the term, Kirschenbaum used the example $W A X W E B$ that included two releases: an online version and a stand-alone CD-ROM. ${ }^{37}$ While this example explained the term, Kirschenbaum did not discuss how a release can vary when the work is distributed across multiple countries by different publishers. This limitation may be due to WAXWEB's limited release: it was not an internationally distributed cultural product with multiple publishers in dozens of countries, like The Last of $U s .{ }^{38}$ As discussed above, each of these

36. To use the network features of a PlayStation console, one is required to create a PlayStation account. This account will provide the user with a unique login identification, but it will also be linked to a specific PS Store. This means that if any purchases are made with that account, the purchases can only be accessed through that account on that PS Store. PlayStation accounts are not transferrable across different PS Stores. In order to access videogames on another PS Store, one would have to create another account.

37. Kirschenbaum, "Editing the Interface," $46-47$

38. While researching this project, release was the most convoluted and challenging concept to examine. I considered the possibility that I may be shoehorning bibliographical information into the concept of release instead of creating several distinct concepts to distinguish product codes, product identifiers, and issue numbers, but I always came back to the initial concept of computationally compatible 


\section{The Last of Us and The Last of Us Remastered}

aspects of a release- product code, product identifier, and issue number-follows Kirschenbaum's definition. This suggests that it is not the concept of release that is at issue but its growing relevance in a global society where cultural products are released in physical and digital formats in numerous countries by multiple publishers following local governmental regulatory policies and publisher practices. In his initial definition, Kirschenbaum suggested that release could be distinguished by its physical format, such as a Blu-ray disc or online-digital download. I would suggest that the concept be further distinguished by publisher and regional releases that further differentiate how a born-digital text or electronic record is functionally integrated as a release of a work within a specific layer and version. ${ }^{39}$ In the case of The Last of $U s$, I examined three distinct release identifiers beyond its physical format: product code, product identifier, and issue number. Further exploration of the term release using other born-digital texts and electronic records may likely reveal additional methods for distinguishing a release. As new platforms and formats are released alongside geo-political shifts and publisher practices, it is likely the term release will become more convoluted than it already is, especially when publishers continue to employ new strategies for tackling piracy and copyright infringement.

Following the layer, version, and release are the object, state, and instance of a videogame. Kirschenbaum defines object as, "a discrete unit of code that consists of both data and instructions for operating on that data." Objects can be the numerous files within a videogame, such as images, videos, animations, audio, and programming code. An analysis of these discrete units, however, is not necessarily pertinent to the discussion of how to describe and cite specific copies of videogames. Such an analysis would be akin to citing a book based on an illustration within the book. However, Kirschenbaum further defines object as a "generic identifier for some discrete digital entity, such as a file (and a file can itself be defined as a named collection of data that persists over

but not functionally integrated. All three features were examples of release but distinguished the concept in distinct bibliographic terminology.

39. It should be noted, that while these additional distinctions for the term release are useful in the case of The Last of Us, further application of the term to other born-digital texts and electronic records may yield additional concepts for defining a release. 
time)." ${ }^{40}$ As such, while specific units of videogames can be considered objects, so can much broader digital entities, such as executable files. Executable files are compiled code that performs tasks for a software program to function. In the case of videogames, they run all the numerous discrete objects so a player can play through a videogame. Much like how pages guide a reader's advance through the story of a literary text, the executable file allows players to progress through a videogame. The executable file, therefore, for the purposes of citation, is the object that represents the videogame.

If the object is the file, then the state is the computational composition of that file in its data format. Much like how stereotype plates can be imposed in different arrangements to print in different bibliographical formats, file formats can be variably structured at the computational level. For Blu-ray disc copies of The Last of $U s$ on the $\mathrm{PS}_{3}$, the executable file is in a BIN format. BIN files are compressed binary files that include instructions for how to run the videogame software. On $\mathrm{PS}_{3}$ copies of The Last of $U_{s}$ the BIN files contain approximately $20-30 \mathrm{MB}$ of data that include instructions for how to locate and grab other objects on the Blu-ray disc for any given task. As such, when one inserts the videogame into a Blu-ray disc reader, one can view all the objects for The Last of Us. However, The Last of Us digital download and The Last of Us Remastered Blu-ray disc and digital download are in PKG format. ${ }^{41}$ PKG files are installers for software applications. As such, when you download the digital file or insert the Blu-ray disc, the PKG file is installed onto the $\mathrm{PS}_{3}$ or $\mathrm{PS}_{4}$ console with all of the objects required to play the videogame. This means that when we try to view the objects on a $\mathrm{PS}_{4}$ copy of The Last of Us Remastered, the only file visible is the PKG file. This transition to PKG file application installers also explains why the $\mathrm{PS}_{3} \mathrm{Blu}$-ray discs and digital downloads have different release codes while the $\mathrm{PS}_{4}$ Blu-ray discs and digital downloads have the same release codes; they have the same codes because they are the same file format. Even though we cannot access all objects without breaking DRMs and reverse engineering the compiled code of the executable files, there are

40. Kirschenbaum, "Editing the Interface," 47-48.

41. After much research in $\mathrm{PS}_{3}$ and $\mathrm{PS}_{4}$ developer wikis and forums, it became evident that all $\mathrm{PS}_{3}$ Blu-ray discs contained BIN executable files, and all $\mathrm{PS}_{3}$ and $\mathrm{PS}_{4}$ digital downloads and $\mathrm{PS}_{4}$ Blu-ray discs contained PKG files. 


\section{The Last of Us and The Last of Us Remastered 481}

still methods of deduction through computational and paratextual evidence that show the bibliographical variants and similarities among releases of videogame editions.

The instance refers to, "a particular object in a particular state as presented in a particular software environment." ${ }^{42}$ This is essentially the software environment that allows the videogame's executable file to run. In the context of The Last of $U s$ editions, they run on the software environments of the $\mathrm{PS}_{3}$ and $\mathrm{PS}_{4}$, respectively. However, videogame consoles, like most platform environments, are constantly updated with firmware updates to fix bugs, enhance current features, or include new content for users. ${ }^{43}$ The $\mathrm{PS}_{3}$ and $\mathrm{PS}_{4}$ constantly receive software updates, which means changes to the core gameplay hardware and software, such as the dual-shock controller, can considerably affect how a player experiences the game. While there are currently no identified issues for any of the previous software updates for the $\mathrm{PS}_{3}$ and $\mathrm{PS}_{4}$ that affect the gameplay of The Last of $U$ s editions, it is always possible that future updates to those consoles could alter gameplay. ${ }^{44}$

\section{IV}

Now that we have identified the six core bibliographical features for a description of The Last of Us editions in the context of Sony Computer Entertainment development, publishing, and manufacturing practices, we can now cite a specific copy. According to Kirschenbaum, "a copy refers to one precise and particular instance of one particular state of an object." 45 For example, if I purchase a digital-download release of The Last of $U_{s}$ on the United States PS Store for the PS 3 layer, that copy will be playable as a particular version in a particular instance of the $\mathrm{PS}_{3}$ software environment as a PKG state of the executable file object.

42. Kirschenbaum, "Editing the Interface," 48.

43. Firmware versions can be located in the $\mathrm{PS}_{3}$ or $\mathrm{PS}_{4}$ systems information sections of the console as "system software version."

44. Issues with gameplay are usually identified through breaks in the videogame where players are having difficulty progressing because of sluggish firmware updates to a console's operating system or controller. So while there are no clearly defined "breaks," as observed with The Last of Us editions in online forums, that does not mean gameplay has not been affected in subtle and nuanced ways.

45. Kirschenbaum, "Editing the Interface," 49. 


\section{$482 \quad$ Bibliographical Society of America}

As discussed throughout this paper, all of this information is relatively accessible through legal means.

A template description of a videogame might read:

Title. Platform and Version Number (Executable File Format). Product Code, Issue Number (Place of Distribution). Product Identifier [Media Specifications]. Developer: Publisher, Publication Data. Software Version and Download Source.

The structure of the citation follows similar citation practices for other textual formats, including the author (developer), title, publisher, and publication date, but also includes the layer with the firmware version, the version number, the release product code, identifier and issue number, the format specifications for the Blu-ray disc and the PS Store, and the URL of the digital download file.

Applying that template, here are sample descriptions of The Last of $U_{s}$ and The Last of Us Remastered released on the $\mathrm{PS}_{3}$ and $\mathrm{PS}_{4}$ as Bluray discs and digital downloads:

The Last of Us. PS 3 v4.78 (BIN). BCUS-98174, 99146 (Canada). BPDL-453050 [Dual-Layer | 50 GB | H.264/MPEG-4 AVC | 64 KB ECC | 405 NM]. Naughty Dog, Inc.: Sony Computer Entertainment America LLC, 14 June 2013. PSN v1.11.

The Last of Us Remastered. PS 4 v3.15 (PKG). CUSA-00552, 3000288 (Canada). B4DL-002790 [Dual-Layer | 50 GB | H.264/MPEG-4 AVC | 64 KB ECC | 405 NM]. Naughty Dog, Inc.: Sony Computer Entertainment America LLC, 29 July 2014. PSN vi.o6.

The Last of Us. PS 3 v4.78 (PKG). NPUA-8096o (PSN Canada | English). UP9ooo-NPUA80960_oo-THELASTOFUSDIGo. NaughtyDog,Inc.: Sony Computer Entertainment America LLC, 14 June 2013. PSN vi.11 <https://store .playstation.com/\#!/en-ca/games/the-last-of-us/cid=UP9ooo-NPUA80960 _oo-THELASTOFUSDIGoı>

The Last of Us Remastered. PS4 v3.15 (PKG). CUSA-00552 (PSN Canada | English). UP9ooo-CUSAoo552_oo-THELASTOFUSooooo. Naughty Dog, Inc.: Sony Computer Entertainment America LLC, 29 July 2014. PSN vı.o6 $<$ https://store.playstation.com/\#!/en-ca/games/the-last-of-us-remastered -/cid=UP9ooo-CUSAoo552_oo-THELASTOFUSooooo>

While these videogame citations are descriptive, they are not definitive. It is important that we develop a discussion around the citation and bibliographic description of videogames and other born-digital texts 


\section{The Last of Us and The Last of Us Remastered 483}

and electronic records. Current citations of videogames are limited to information on the title, platform layer, developer, publisher, and publication date, without any further detail as to the layer, version, release, object, state, and instance of the copy referenced. The purpose of this paper is to emphasize the considerable variants that exist when within only two editions of a videogame. The vast majority of videogames are available on multiple platforms simultaneously, including the PC, Xbox, PlayStation, Nintendo, as well as on mobile platforms, which further complicates the potential variance a researcher faces when describing a videogame copy. Each new software patch to the videogame and

firmware update to the platform environment alters the videogame in some computational instance. Consequently, the gameplay a scholar references in a publication may not match that of the reader attempting to verify the findings, because they are playing a different layer, version, release, object, state, and instance of the videogame.

While my research has uncovered some of the internal workings of Sony Computer Entertainment, its publishing and distribution practices are embedded in a secretive industry hidden behind DRMs and legal procedures designed to protect their intellectual property, copyright, and trademark entities. The information behind the creation and allocation of release product codes, identifiers, and issue numbers is currently almost as inaccessible as the publishing practices of a fifteenth-century printing house. Sony Computer Entertainment does not publically release information about its publishing and manufacturing practices. This is not unexpected, because videogame publishers and console manufacturers want to limit the abilities of crackers, hackers, and modders to alter their content for their own distribution. Ironically, however, almost all of this research relied on those same developers, hackers, and modders who shared their knowledge of Sony Computer Entertainment's practices on wikis and public forums. Even so, a lot of this research project involved purchasing multiple copies of The Last of $U s$ editions and scouring online marketplaces around the world to view the digital images e-sellers had posted of their new and used copies of The Last of Us and The Last of Us Remastered. In many ways, my research involved developing a mini-Short-Title-Catalogue to determine how many potential variant copies were in circulation around the world as Blu-ray discs and digital downloads on PS Stores. Even with this accumulated information, it is still not clear that I have accounted for all 


\section{$484 \quad$ Bibliographical Society of America}

authorized and commercially released editions. While searching online marketplaces I discovered early press releases of The Last of Us editions that suggest there are other extant forms of the game, such as unfinished Beta versions. ${ }^{46}$ Further, this paper does not account for the numerous pirated copies in existence that are emulated through different platform environments.

The bibliography of videogames is a field nuanced by secretive industry practices that embed the provenance of variants behind computational mechanisms and legal protections. The publishing history of The Last of $U_{s}$ trademark, while a significant release in the short history of videogames, is unremarkable in the bibliographic sense because there are a limited number of variant copies to research. Though this aspect of The Last of $U s$ editions made it an ideal case study for exploring the basic features of physical and digital releases of the videogame, it also revealed many of the hidden publishing and manufacturing practices of Sony Computer Entertainment for identifying specific copies of $\mathrm{PS}_{3}$ and $\mathrm{PS}_{4}$ videogames. Subsequent, and more interesting, studies in the bibliography of videogames will likely look at the variants between editions released by different developers and publishers, the publishing and manufacturing practices between platforms, the complicated version history of a particular videogame, or the convoluted releases of a particular title. Such studies may note bibliographic information that was not accounted for in this study. However, we are still at the early stages of describing and classifying born-digital texts and electronic records. It will be through further case studies and in-depth developer and publisher histories that scholars will begin to game the computational and legal system to progress to the next level of bibliographical challenges. ${ }^{47}$

46. Beta versions are pre-releases of videogames usually distributed to the press in advance for review or to testers to locate bugs for software patches before an official commercial release.

47. An earlier version of this article was delivered at the 2016 Bibliographical Society of America's annual meeting. I would like to thank my supervisor, Sara Grimes, and Alan Galey, from the University of Toronto, for their feedback on earlier versions of this article. 